\title{
Radiopacidad de Cementos de Resina Compuesta Evaluados con Técnica Radiográfica Digital.
}

\section{Radiopacity of Resin Cements Evaluated with Digital Radiographic Technique.}

\author{
Paulo Gallardo ${ }^{1}$, Camila Corral-Nuñez ${ }^{1 *}$, Sylvia Osorio ${ }^{1,2}$, Juan Estay ${ }^{1,2}$
}

\begin{abstract}
1. Departamento de Odontología Restauradora, Facultad de Odontología, Universidad de Chile, Chile.

2. Departamento de Patología y Medicina Oral, Facultad de Odontología, Universidad de Chile, Chile.

* Correspondencia autor. Camila Corral Núñez | Teléfono: +56 229781742 | E-mail: camila. corral@odontologia.uchile.cl.| Departamento de Odontología Restauradora, Facultad de Odontología, Universidad de Chile, Chile. Trabajo recibido el 04/01/2019.

Aprobado para su publicación el 17/03/2019
\end{abstract}

\begin{abstract}
RESUMEN
Objetivo: comparar la radiopacidad de distintos cementos de resina y corroborar si cumplen con los requisitos de normativas ISO. Material y método: Se prepararon muestras de $1 \mathrm{~mm}$ de grosor de los cementos de resina: RelyX U200 (3M ESPE), Calibra (Dentsply Sirona), Calibra Universal (Dentsply Sirona) y Bifix SE (VOCO). Las muestras fueron radiografiadas junto a una cuña de aluminio escalonada. Las imágenes fueron analizadas para determinar el valor en escala de grises y su respectivo valor en $\mathrm{mm}$ de Al. Los datos fueron analizados para comparar la radiopacidad de los materiales entre sí y con los requisitos establecidos por normas ISO. Resultados: La radiopacidad equivalente en $\mathrm{mm}$ de $\mathrm{Al}$ en orden descendiente fue de 2,84 $\pm 0,18$ para Calibra, 2,37 $\pm 0,15$ para Bifix SE, 2,26 $\pm 0,14$ para Calibra Universal y de $1,67 \pm 0,07$ para RelyX U200. Estos valores son todos mayores al mismo grosor de aluminio exigido por la norma ISO 4049. Se establecieron diferencias estadísticamente significativas entre los cementos. Conclusiones: Se determinó que existen diferencias en la radiopacidad entre los cementos de resina compuesta estudiados. Sin embargo, todos los cementos evaluados cumplen con la norma ISO4049 de radiopacidad
\end{abstract}

PALABRAS CLAVE:

Cemento de resina; Materiales dentales; Radiografía dental digital; Radiopacidad.

Rev. Clin. Periodoncia Implantol. Rehabil. Oral Vol. 12(2); 77-80, 2019.

\section{ABSTRACT}

Objective: Compare the radiopacity of different resin cements and verify their compliance with the requirements of ISO regulations. Material and method: Samples $-10 \mathrm{~mm}$ in diameter and $1 \mathrm{~mm}$ thickness- of the resin cements RelyX U200 (3M ESPE), Calibra (Dentsply Sirona), Universal Calibra (Dentsply Sirona) and Bifix SE (VOCO) were prepared. Radiographies of the samples with a stepped aluminum wedge were obtained. For each sample, the grayscale and $\mathrm{mm}$ of Al values were calculated. The data were analyzed to compare the radiopacity of the materials and to contrast them with the requirements established by ISO standards. Results: The equivalent radiopacity in $\mathrm{mm}$ of $\mathrm{Al}$ in descending order was $2.84 \pm 0.18$ for Calibra, $2.37 \pm 0.15$ for Bifix SE, $2.26 \pm 0.14$ for Calibra Universal and $1.67 \pm 0.07$ for RelyX U200. These values exceed the value required by ISO 4049 standard. Statistically significant differences were established between the cements. Conclusion: There are differences in the radiopacity of the resin cements studied. However, all the cements complied with ISO 4049 radiopacity standards.

KEY WORDS:

Dental digital radiography; Dental materials; Resin cement, Radiopacity.

Rev. Clin. Periodoncia Implantol. Rehabil. Oral Vol. 12(2); 77-80, 2019.

\section{INTRODUCCIÓN}

Los cementos de resina compuesta son materiales usados para la cementación adhesiva de diversas restauraciones indirectas, tales como coronas, prótesis fija plural, carillas, prótesis implanto-soportadas, espigas radiculares e incrustaciones ${ }^{(1,2)}$. Debido al incremento en el uso de restauraciones estéticas, estos cementos han emergido fuertemente en la práctica odontológica en los últimos años ${ }^{(3)}$. Presentan ventajas cuando se les compara con los cementos convencionales, dentro de ellas una mayor resistencia a la carga oclusal y a la fractura en materiales de recubrimiento cerámico, y una menor microfiltración ${ }^{(2)}$.

La radiopacidad es una propiedad fundamental en los materiales de cementación, que permite pesquisar la línea de cementación entre diente y restauración, y detectar presencia de excesos del material cementante, caries adyacente a la restauración y evaluar adaptación marginal(1-3). La cantidad y tipo de relleno radiopaco, y el número atómico de las partículas de relleno inorgánico influyen en la radiopacidad de los cementos de resina ${ }^{(1,4,5)}$. Esta condición debe considerarse adecuadamente en los cementos de resina, debido a que los agentes radiopacificadores incrementan la 
expansión térmica del cemento e hidrolizan silanos, pudiendo aumentar el nivel de opacidad de los materiales e inestabilidad del $\operatorname{color}^{(6)}$.

Estudios que evalúen la radiopacidad de cementos de resina compuesta son escasos, más aún en relación a materiales de reciente aparición en el mercado ${ }^{(2,3)}$. Es así como, el objetivo de este estudio fue determinar el valor de radiopacidad de cementos de resina compuesta disponibles en el mercado, usados para cementación adhesiva. La hipótesis del presente estudio es que existen diferencias entre los valores de radiopacidad de los distintos cementos de resina estudiados, y que estos son mayores al requisito establecido por la norma ISO 4049.

\section{MATERIAL Y MÉTODO}

\section{Preparación de los cuerpos de prueba}

Este estudio evaluó la radiopacidad de cuatro cementos de resina compuesta y dos cementos de referencia, de vidrio ionómero y de fosfato de zinc (Tabla 1). Se prepararon los materiales siguiendo las indicaciones de la norma ISO 4049. Se confeccionaron 5 discos de $10 \pm 0,1 \mathrm{~mm}$ de diámetro y de $1 \pm 0,1 \mathrm{~mm}$ de espesor, utilizando un molde diseñado e impreso en 3D para asegurar el grosor adecuado de las muestras. Posterior a su polimerización, las muestras se mantuvieron en una estufa a $37 \pm$ $0,2{ }^{\circ} \mathrm{C}$ (Estufa UN30, Memmert) por 24 horas, a la espera de su completo endurecimiento. El grosor de las muestras confeccionadas se corroboró con un pie de metro digital (Pie de metro e5001002, Veto, Chile, precisión $0,01 \mathrm{~mm}$ ), y se seleccionaron solo aquellos que presentaban un grosor de $1 \pm 0,1 \mathrm{~mm}$. Los cuerpos fueron radiografiados de manera preliminar (Equipo de rayos X Myray RXAC, Imola, Italia). Las radiografías fueron estudiadas por dos especialistas en Radiología Maxilofacial, descartándose aquellas muestras que presentaron burbujas o defectos, hasta obtener cinco muestras por cada material.

\section{Obtención de la imagen radiográfica}

La imagen radiográfica se obtuvo posicionando las muestras, de cada grupo de material, junto con una cuña de aluminio escalonada de 1 a 10 $\mathrm{mm}$, con una pureza mayor a 99\% de aluminio (OMT160, Odeme, Santa Catarina, Brasil). Se utilizó una película de fósforo fotoestimulante (PSP, Photostimulable Phosphor Plate) oclusal, tamaño 48x54 mm, FireCR Dental (3DISC Corp., Daejeon, Korea) (Figura 1). La toma radiográfica se estandarizó acorde a la normativa ISO 4049. La distancia focal se fijó en $400 \mathrm{~mm}$ y el tiempo de exposición en 0,4 segundos. El equipo a utilizar fue MyRay RXAC (Imola, Italia), $70 \mathrm{Kv}$ y $8 \mathrm{~mA}$. La toma radiográfica se realizó por un solo operador, odontólogo, especialista en Radiología Maxilofacial. La PSP expuesta fue procesada en el dispositivo FireCR Dental Reader (3DISC Imaging. Virginia, USA) e importada al software Planmeca Romexis 4.4.0.R (Planmeca Helsinki, Finlandia). Posteriormente la imagen se exportó en formato tiff sin compresión ni filtros de mejoramiento. Los archivos tiff fueron utilizados mediante el programa Adobe Photoshop CC (Adobe, California, USA). En cada imagen se determinó el valor, en escala de grises, de cada segmento de la cuña escalonada de aluminio (área de $100 \times 500$ pixeles), así como cada una de las 5 muestras. Esto se realizó seleccionando un área representativa (área de $200 \times 100$ pixeles) del objeto de análisis y utilizando la herramienta Histograma, la cual determinó de manera automática, el promedio de valor de gris de todos los pixeles incluidos en el área previamente seleccionada. Este valor puede variar desde 0 , que corresponde a negro puro, hasta 255 que representa blanco puro.

\section{Análisis estadístico}

Los datos de valor de gris obtenidos se tabularon en el programa Microsoft Excel 2016 (Microsoft, Washington, USA). Luego se graficó la curva "Milímetros de Aluminio/Valor en Escala de Grises" y, mediante ésta,

Tabla 1. Caracterización de los cementos en estudio de acuerdo a fabricante, composición y tipo de relleno y/o radiopacificador, de acuerdo a información declarada por los fabricantes.

\begin{tabular}{|c|c|c|c|c|}
\hline Material / Color & Fabricante & Tipo de cemento & Composición & $\begin{array}{c}\text { Relleno / } \\
\text { Radiopacificador }\end{array}$ \\
\hline RelyX U200 / A30 & $\begin{array}{c}\text { 3M ESPE, St. Paul, } \\
\text { MN, USA }\end{array}$ & $\begin{array}{c}\text { Cemento resina } \\
\text { compuesta, curado } \\
\text { dual }\end{array}$ & $\begin{array}{l}\text { Pasta base: fibra de vidrio, ésteres de } \\
\text { ácidos fosfóricos metacrilados, TEGDMA, } \\
\text { sílice silano, persulfato de sodio } \\
\text { Pasta catalizadora: fibra de vidrio, } \\
\text { dimetacrilatos, sílice silano, p-toluensulfinato } \\
\text { de sodio, hidróxido de calcio }\end{array}$ & $\begin{array}{l}43 \% \text { en vol. / no } \\
\text { informado }\end{array}$ \\
\hline Calibra / Light & $\begin{array}{l}\text { Dentsply, Milford, DE, } \\
\text { USA }\end{array}$ & $\begin{array}{c}\text { Cemento resina } \\
\text { compuesta, curado } \\
\text { dual }\end{array}$ & $\begin{array}{l}\text { Pasta base: bis-GMA, vidrio de borato } \\
\text { de bario fluoroaluminosilicato, resina de } \\
\text { dimetacrilato polimerizable, dióxido de } \\
\text { titanio, sílica hidrofóbica amorfa } \\
\text { Pasta catalizadora: mismos componentes } \\
\text { que la base, peróxido de benzoilo }\end{array}$ & $\begin{array}{l}\text { No informado / } \\
\text { Dióxido de titanio } \\
\text { Vidrio de } \\
\text { borato de bario } \\
\text { fluoroaluminosilicato }\end{array}$ \\
\hline $\begin{array}{c}\text { Calibra Universal / } \\
\text { Ligth }\end{array}$ & $\begin{array}{c}\text { Dentsply, Milford, DE, } \\
\text { USA }\end{array}$ & $\begin{array}{l}\text { Cemento resina } \\
\text { compuesta, curado } \\
\text { dual }\end{array}$ & $\begin{array}{l}\text { Dimetacrilato de Uretano, resinas } \\
\text { de di- y tri-metacrilato, HEMA, resinas } \\
\text { de acrilato modificadas con ácido } \\
\text { fosfórico, vidrio de borato de bario } \\
\text { fluoroaluminosilicato, iniciador orgánico de } \\
\text { peróxido, canforoquinona, óxido de fosfeno, } \\
\text { hidroxitolueno butilado, dióxido de titanio, } \\
\text { óxido de silicio, óxio de hierro }\end{array}$ & $\begin{array}{l}48,7 \% \text { en vol / } \\
\text { Dióxido de } \\
\text { titanio, vidrio de } \\
\text { borato de bario } \\
\text { fluoroaluminosilicato }\end{array}$ \\
\hline
\end{tabular}

Bifix SE / Universal $\begin{array}{cc}\text { Voco } \mathrm{GmbH}, & \begin{array}{c}\text { Cemento de resina } \\ \text { cuxhaven, Alemania }\end{array} \\ \begin{array}{c}\text { compuesta, curado } \\ \text { dual }\end{array}\end{array}$

Bis-GMA, UDMA, glicerol dimetacrilato, monómeros fosfatados, iniciadores, estabilizadores
$70 \%$ en peso y $45 \%$ en vol. / no informado

\author{
Cemento vidrio \\ ionómero \\ convencional
}

$\begin{array}{ccc}\text { uji GC Gold Label / } & \text { GC America Inc., } & \text { ionómero } \\ \text { Ligth yellow } & \text { Illinois, USA } & \text { convencional }\end{array}$

Goldsmith Fosfato
de Zinc / - $\quad$ Goldsmith, USA $\quad \begin{gathered}\text { Cemento fosfato de } \\ \text { zinc }\end{gathered}$




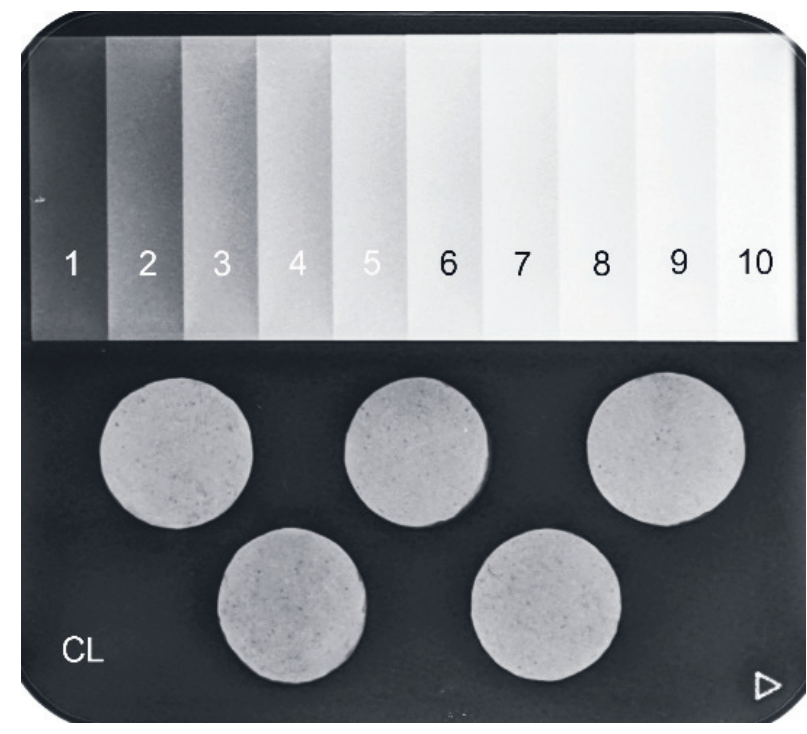

Figura 1. Radiografía representativa de las muestras de cemento de resina (Calibra) con la cuña de aluminio escalonada

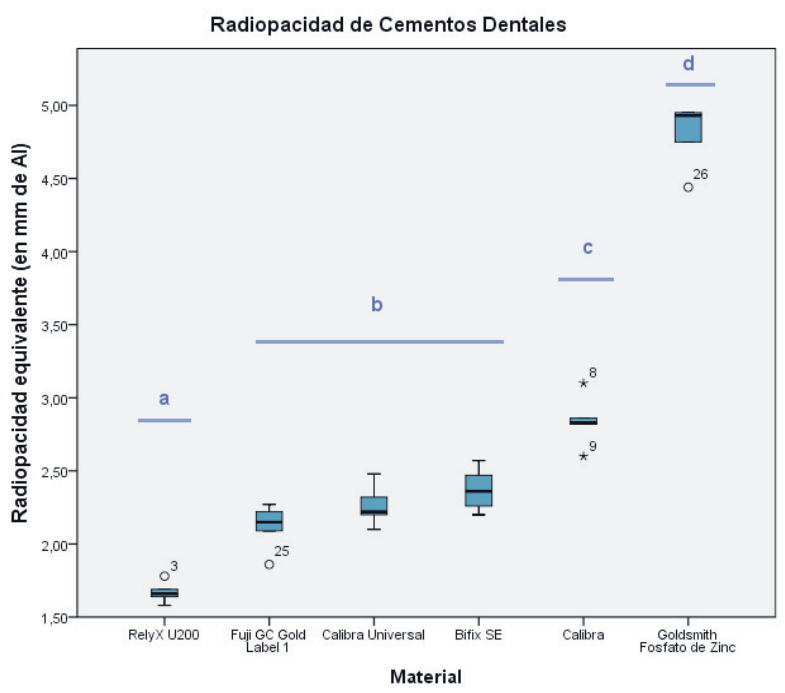

Figura 2. Radiopacidad equivalente en $\mathrm{mm}$ de $\mathrm{Al}$ de los diferentes cementos de resina compuesta. Cementos con distintas letras representan diferencias significativas $(p<0.05)$

se determinó la radiopacidad equivalente, en milímetros de aluminio, del valor de gris de cada una de las muestras a partir de la ecuación logarítmica entregada por la curva calculada con Microsoft Excel 2016 (Microsoft, Washington, USA).

Los datos de radiopacidad equivalente de las muestras de cada grupo, expresada en milímetros de aluminio, se procesaron a través del software SPSS Statistics 21.0 (IBM, New York, USA). Se realizó estadística descriptiva de cada grupo, presentando los resultados como medias desviación estándar, y se corroboró si estos cumplían con los requisitos de radiopacidad mínima exigidos por la norma ISO 4049: "Materiales restauradores, de relleno y cementación, basados en polímeros". A continuación, se utilizó test de Shapiro-Wilk para determinar la normalidad de la distribución de los datos. Finalmente, una vez que se determinó que la distribución era normal, los datos entre grupos se analizaron con ANOVA de un factor y post-hoc HSD Turkey, con un nivel de significancia de $p=0,05$.

\section{RESULTADOS}

Los valores de radiopacidad media de los cementos de resina compuesta estudiados estuvieron en el rango de 1,67 a 2,84 $\mathrm{mm}$ de Al. De acuerdo a resultado de test Shapiro-Wilk los valores de radiopacidad de los cementos estudiados presentan una distribución normal. Test ANOVA reveló que existen diferencias estadísticamente significativas entre los grupos estudiados $(p<0,001)$, determinándose por medio del test post hoc HSD Tukey, tres subconjuntos de cementos de resina con diferencias estadísticamente significativas $(p<0,05)$ (Figura 2). El subconjunto "a" lo constituye RelyX U200, con el menor valor de radiopacidad media $(1,67$ $+/-0,07)$. Calibra Universal y Bifix SE conforman el subconjunto "b" $(2,26$ $+/-0,14$ y $2,37+/-0,15$ respectivamente), con valores de radiopacidad intermedios. Calibra corresponde al subconjunto "c", con el mayor valor de radiopacidad media $(2,84+/-0,18)$. Los cementos de referencia, Fuji GC Gold Label 1 presentaron un valor de radiopacidad estadísticamente similar al subconjunto "b" $(2,12+/-0,16)$, y Goldsmith Fosfato de Zinc obtuvo el mayor valor de los cementos estudiados, conformando el subconjunto "d" $(4,80+/-0,22)$

\section{DISCUSIÓN}

La radiopacidad es una propiedad fundamental en los cementos de resina compuesta usados en odontología, permitiendo detectar caries secundaria, adaptación marginal de la restauración cementada, excesos del material cementante y evaluar la interfaz entre diente y restauración ${ }^{(3,4)}$. Es por esto, que este estudio experimental determinó y comparó la radiopacidad de cuatro cementos de resina compuesta disponibles en el mercado: RelyX U200, Calibra, Calibra Universal y Bifix SE, comparándolos con cementos de indicaciones similares, ampliamente estudiados cuando a esta propiedad se refiere. Los resultados obtenidos demuestran que existen diferencias significativas en la radiopacidad de los cementos de resina compuesta estudiados. Se demuestra, además, que todos los cementos estudiados cumplen lo exigido por la norma ISO 4049, con valores de radiopacidad equivalente de al menos el mismo grosor de aluminio(7).

La radiopacidad media obtenida en este estudio para Calibra fue la mayor de los cementos de resina estudiados $(2,84 \pm 0,18 \mathrm{~mm}$ de Al). En relación al antecedente reportado sobre su valor de radiopacidad, Montes-Fariza y cols. reportaron un valor de radiopacidad de $3,51 \mathrm{~mm}$ de Al ${ }^{(8)}$. Esta diferencia puede deberse a distinta metodología en la obtención de las muestras a radiografiar. Montes-Fariza y cols. obtienen su valor de radiopacidad para Calibra a partir de una única muestra de $1 \mathrm{~mm}$ de espesor, distinto a las cinco muestras usadas para este cemento en el presente estudio. Es de considerar al respecto, que la mezcla manual de dos pastas incorpora burbujas de aire que inhiben la polimerización debido a la presencia de oxígeno, afectando el grado de conversión del cemento, pudiendo provocar diferencias de valores de radiopacidad en distintas muestras ${ }^{(9-11)}$

RelyX U200 presentó la menor radiopacidad media obtenida en el presente estudio $(1,67 \pm 0,07 \mathrm{~mm}$ de $\mathrm{Al})$. El único estudio disponible que evaluó esta propiedad en este cemento reportó un valor de radiopacidad entre 1,96 a $2,46 \mathrm{~mm}$ de $\mathrm{Al}^{(2)}$. Las diferencias en los resultados obtenidos entre este experimento y el estudio disponible se pueden explicar en el tipo de material usado. Dukic utiliza el cemento RelyX U200 con dispensador de automezcla (RelyX U200 Automix), en contraste al usado en este estudio, con mezcla manual (RelyX U200 Clicker). Como se mencionó previamente, la mezcla manual de dos pastas incorpora burbujas de aire, las que se disminuyen cuando se usan jeringas de automezcla ${ }^{(9-11)}$.

Los cementos Calibra Universal y Bifix SE presentaron valores de radiopacidad intermedios, sin diferencias estadísticamente significativas entre ellos (2,26 $\pm 0,14$ y $2,37 \pm 0,15 \mathrm{~mm}$ de Al respectivamente). Sólo existe un estudio previo que evalúa la radiopacidad de Bifix $\mathrm{SE}$, con valores que oscilan entre 2,27 y $2,79 \mathrm{~mm}$ de Al, resultados similares a los obtenidos en la presente investigación ${ }^{(2)}$. En el caso de Calibra Universal, no se reportan estudios que evalúen su radiopacidad, considerando que es un cemento de reciente aparición en el mercado.

Si bien es cierto, todos los cementos de resina estudiados presentan valores de radiopacidad por sobre lo que establece la norma ISO 4049, es decir, mayor a la radiopacidad equivalente de la dentina, hay autores que difieren sobre este estándar, al indicar que esta radiopacidad debiera tener relación con los tejidos sobre los que se va a usar el cemento, esto es en dentina y/o esmalte ${ }^{(1,12)}$. Según reportes de radiopacidad equivalente de esmalte, ésta oscila entre 1,58 y $2,33 \mathrm{~mm}$ de $\mathrm{Al}^{(2,3,4,8,8,13)}$. Al respecto, un estudio reciente de Hosney y cols., establece que, al cementar bloques cerámicos, que presentan radiopacidad cercana a la dentina, la línea de cementación es claramente detectable radiográficamente cuando el cemento a utilizar presenta valores de radiopacidad mayor a la de esmalte, contrario a lo que ocurre cuando se usa un cemento de radiopacidad de $1,25 \mathrm{~mm}$ de $\mathrm{Al}^{(3,14)}$. Por lo que en casos que la línea de cementación se presente en esmalte, en concordancia a lo obtenido en este estudio, el valor de radiopacidad media obtenida para RelyX U200 dificultaría la correcta identificación de la línea de cementación, de excesos del material cementante, de un diagnóstico adecuado de caries secundaria y de la adaptación marginal.

La radiopacidad en los cementos de resina compuesta está determinada por la presencia de elementos radiopacificadores y cantidad de relleno inorgánico en su composición. Se ha establecido que el tipo, la 
concentración y el tamaño de la partícula de radiopacificador afectan el valor de radiopacidad de los distintos materiales dentales ${ }^{(4,15)}$. Se ha reportado que estos elementos afectan las propiedades estéticas de los cementos de resina, tales como la estabilidad del color o el nivel de opacidad, lo que no es deseable considerando las indicaciones de estos cementos ${ }^{(6,12)}$. RelyX U200 presenta un relleno radiopaco de $43 \%$ en volumen, lo que explicaría en parte su menor valor de radiopacidad ${ }^{(16)}$. Se ha reportado que la radiopacidad de los materiales dentales es inferior a la del esmalte cuando el relleno inorgánico que presentan es menor al $70 \%$ en volumen ${ }^{(17)}$. Calibra tiene como agente radiopacificador su contenido de titanio, que tiene un alto número atómico, lo que explica su alto valor de radiopacidad obtenido cuando se le compara con los otros cementos estudiados ${ }^{(5,18)}$. Para Calibra Universal y Bifix SE, sus altos porcentajes de relleno inorgánico que, según sus fabricantes ascienden a $48,7 \%$ en volumen, y al $70 \%$ en peso y $45 \%$ en volumen respectivamente, sumado a la presencia de titanio en Calibra Universal, actuarían como agentes radiopacificadores. Se requieren entonces, más estudios que evalúen el incremento en el contenido de relleno inorgánico o de agentes radiopacificadores, $\sin$ alterar las propiedades físicas y estéticas de los cementos de resina compuesta, así como, la contribución en la radiopacidad, de los tipos de matriz usada en los diversos cementos, tales como HEMA, UDMA, 10-MDP, Bis-GMA y TEGDMA ${ }^{(12)}$.

Considerando que todos los cementos de resina estudiados tienen como indicación la cementación de espigas prefabricadas intrarradiculares, se hace necesario que el valor de radiopacidad que presentan sea superior 0 por lo menos cercano al que establece la norma ISO 6876: "Materiales de sellado del canal radicular", de $3 \mathrm{~mm}$ de $\mathrm{Al}$, aunque estos materiales no se incluyan en esta norma ${ }^{(19)}$. Se ha reportado en un estudio ex vivo que, al cementar espigas prefabricadas de diferentes composiciones, aumenta la radiopacidad de la estructura fijada cuando se usan cementos de resina con mayor relleno inorgánico ( $70 \%$ en peso), en comparación con el uso de un cemento de menor relleno ( $30 \%$ en peso). Desde un punto de vista clínico, es relevante considerar, sin embargo, que cuando espigas menos radiopacas son usadas en combinación con cementos de menor relleno inorgánico, el poste es aún distinguible radiográficamente de las estructuras radiculares que lo rodean ${ }^{(20)}$. Debido a lo anterior, es díficil determinar la radiopacidad adecuada para cada aplicación clínica, sin embargo concordamos con Junqueira et al. ${ }^{(21)}$ en que la radiopacidad de un material debería ser moderada, levemente superior a la densidad de la dentina, radiopacidades muy elevadas (similares a densidad metálica) podrían dificultar la correcta visualización de los límites del material o su homogeneidad interna. Lo anterior sugiere que exigencias específicas de radiopacidad sean requeridas para estos materiales de uso endodóntico, y que más estudios evalúen esta propiedad en el uso conjunto de espigas y material de cementación.

En el presente estudio se incluyeron también dos cementos de referencia, de materiales usados para cementación fosfato de zinc (Goldsmith Fosfato de Zinc) y vidrio ionómero (Fuji GC Gold Label 1), los cuales presentaron valores de radiopacidad similares a los reportados en la literatura(4), lo que valida los resultados obtenidos en el presente estudio. Goldsmith Fosfato de Zinc fue el cemento con mayor valor de radiopacidad de los evaluados, cercano a $5 \mathrm{~mm}$ de Al. En el caso del cemento de vidrio ionómero evaluado, este presentó radiopacidad similar al de otros cementos de resina compuesta evaluados, como Calibra Universal y Bifix SE y fue significativamente mayor a la radiopacidad de Relyx U200. A los cementos de vidrio ionómero, de manera similar que a los de resina compuesta, se les agregan elementos con alto número atómico, como estroncio y bario, para incrementar la radiopacidad de estos ${ }^{(4)}$

Los datos reportados en el presente estudio, no consideran los posibles cambios en la radiopacidad que se podrían generar con el tiempo, lo cual es de interés para los controles futuros de restauraciones cementadas. Se ha reportado una disminución en la radiopacidad de cementos de resina compuesta al ser sometidos a simulaciones de envejecimiento. Lo que se ha explicado por la liberación de componentes que no reaccionan y/o por la degradación de la matriz polimérica en el tiempo ${ }^{(21)}$. Por ello, es relevante considerar la biocompatibilidad de los agentes radiopacos que se agregan y que podrían ser liberados con el tiempo. Con respecto óxidos de agentes radiopacos, como el de bario, se ha cuestionado su biocompatibilidad, a diferencia de otros agentes radiopacos, como el zirconio que es considerado químicamente inerte y biocompatible ${ }^{(22,23)}$.

La radiopacidad de los materiales dentales es una propiedad fundamental en la práctica clínica, debido a que este examen complementario permite realizar diagnósticos con mayor precisión y mejor control de los tratamientos ${ }^{(1,12,24,25)}$. Sin embargo, se han reportado gran variabilidad en los valores de radiopacidad que presentan, esto además favorecido por la constante aparición de nuevos cementos de este tipo. Es por este motivo que, en esta investigación experimental se determinó y comparó la radiopacidad de cementos de resina de actual comercialización, para ser una referencia tanto para el clínico, a la hora de elegir un material de cementación definitiva y controlarlo radiográficamente, como para los fabricantes.

\section{CONCLUSIONES}

Los cementos de resina compuesta estudiados presentan valores de radiopacidad diversos, estableciéndose tres grupos con diferencias significativas. RelyX U200 es el cemento de resina con el menor valor de radiopacidad; Calibra Universal y Bifix SE tienen una radiopacidad equivalente intermedia; y Calibra presenta el mayor valor de radiopacidad de los cementos de resina analizados en este estudio. Todos cumplen la norma ISO 4049, teniendo valores de radiopacidad mayores al equivalente de dentina.

\section{CONFLICTO DE INTERÉS}

Los autores declaran no tener conflicto de interés

\section{Bibliografía}

1. Pekkan G, Ozcan M. Radiopacity of different resin-based and conventional luting cements compared to human and bovine teeth. Dent Mater J. 2012;31(1):68-75.

2. Dukic W. Radiopacity of Composite Luting Cements Using a Digital Technique. J Prosthod. 2017;10:[E-pub ahead of print]. Disponible en: https://onlinelibrary.wiley.com/ doi/full/10.1111/jopr.12578

3. Hosney S, Abouelseoud HK, El-Mowafy O. Radiopacity of Resin Cements Using Digital Radiography. J Esthet Restor Dent. 2017;29(3):215-221.

4. Tsuge T. Radiopacity of conventional, resin-modified glass ionomer, and resin-based luting materials. J Oral Sci. 2009;51(2):223-230

5. Furtos G, Baldea B, Silaghi-Dumitrescu L, Moldovan M, Prejmerean C, Nica L. Influence of inorganic filler content on the radiopacity of dental resin cements. Dent Mater J. 2012;31(2):266-272.

6. Amirouche-Korichi A, Mouzali M, Watts DC. Effects of monomer ratios and highly radiopaque fillers on degree of conversion and shrinkage-strain of dental resin composites. Dent Mater. 2009;25(11):1411-1418

7. International Standardization Organization ISO 4049, Dentistry polimer-based filling, restorative and luting materials. 3 ed. Geneva: ISO. In: 2009.

8. Montes-Fariza R, Monterde-Hernández M, Cabanillas-Casabella C, Pallares-Sabater A. Comparative study of the radiopacity of resin cements used in aesthetic dentistry. $J$ Adv Prosthodont. 2016;8(3):201-206.

9. Pegoraro TA, da Silva NR, Carvalho RM. Cements for use in esthetic dentistry. Dent Clin North Am. 2007;51(2):453-471.

10. Yan YL, Kim YK, Kim KH, Kwon TY. Changes in degree of conversion and microhardness of dental resin cements. Oper Dent. 2010;35(2):203-210.

11. De Souza G, Braga RR, Cesar PF, Lopes GC. Correlation between clinical performance and degree of conversion of resin cements: a literature review. J Appl Oral Sci. 2015;23(4):358-368.

12. Pekkan G. Radiopacity of dental materials: An Overview. Avicenna J Dent Res. 2016;8(2):e36847.

13. An SY, Lee DH, Lee KB. Radiopacity for Contemporary Luting Cements Using Digital Radiography under Various Exposure Conditions. J Prosthodont. 2015;24(8):642-646.
14. Hosney S, Kandil M, El-Mowafy O. Radiopacity of Nonmetallic CAD/CAM Restorative Blocks. Int J Prosthodont. 2016;29(3):271-273.

15. Taira M, Toyooka $\mathrm{H}$, Miyawaki $\mathrm{H}$, Yamaki M. Studies on radiopaque composites containing ZrO2-SiO2 fillers prepared by the sol-gel process. Dent Mater. 1993;9(3):167171.

16. 3M ESPE. RelyX U200: Hoja de seguridad. [actualizado 2012; citado 5.11.2018] Disponible en: https://multimedia.3m.com/mws/mediawebserver?mwsld=SSSSSuUn zu8IZNqMY ZM8tUnv70m17zHvu9|xUb7SSSSSS--

17. Watts $\bar{D}$. Radiopacity vs. composition of some barium and strontium glass composites. J Dent. 1987;15:38-43.

18. Dentsply. Calibra Esthetic Resin Cement Safety Data Sheet EN. [actualizado 27.06.2013; citado 5.11.2018]. Disponible en: https://www.dentsplysirona.com/ content/dam/dentsply/pim/manufacturer/Restorative/Indirect_Restoration/Permanent Cements/Resin Cements/Calibra/Calibra-Calibra-Silane-Oecmgq5-en-1402

19. International Standardization Organization ISO 6876, Dental root canal sealing materials. 2ed. Geneva: ISO. In:2012.

20. Goracci C, Juloski J, Schiavetti R, et al. The influence of cement filler load on the radiopacity of various fibre posts ex vivo. Int Endod J. 2015;48(1):60-67.

21. Junqueira R, Carvalho R, Yamamoto F, Almeida S, Verner F. Evaluation of radiopacity of luting cements submitted to different aging procedures. $\mathrm{J}$ Prosthodont 2018; 27:853-859.

22. Chen YZ, Lü XY, Liu GD. Effects of different radio-opacifying agents on physicochemical and biological properties of a novel root-end filling material. PLoS One. 2018;13(2):e0191123

23. Ermis RB, Yildirim D, Yildiz G, Gormez O. Radiopacity evaluation of contemporary resin composites by digitization of images. Eur J Dent. 2014; 8(3): 342-347. 24. Akerboom H, Kreulen C, Van Amerongen W, Mol A. Radiopacity of posterior composite resins, composite resin luting cements, and glass ionomer lining cements. $J$ Prosthet Dent. 1993;70(4):351-355

25. Pekkan G, Saridag S, Beriat N. Evaluation of the radiopacity of some luting, lining and filling dental cements. CDR. 2011;35:2-9. 